\title{
Experimental study of crushing process of the crushed stone
}

\author{
Lola Akobirova $^{1 *}$, Karim Gafurov ${ }^{1}$, Jura Jumayev $^{2}$, Firuza Kuldasheva ${ }^{1}$, Doniyor \\ Xikmatov $^{1}$ \\ ${ }^{1}$ Bukhara Engineering Technological Institute, Bukhara, Uzbekistan \\ ${ }^{2}$ Bukhara State University, Bukhara, Uzbekistan
}

\begin{abstract}
Currently, crushers operating in a central impact manner have become widely used, which has several advantages over roller, cone, and cheek crushers. The present work shows the device and principle of a laboratory centrifugal vertical impact crusher for studying the process of crushing local gravel to obtain crushed stone with dimensions of 5-20 $\mathrm{mm}$. The main influencing factors for the process are the average size of the incoming raw materials, the rotation speed of the crusher rotor, and the distance between the accelerator and the annular platform. The output parameter is the concentration of crushed stone with a size of $5-20 \mathrm{~mm}$ in the output (\%). A full factor experiment $\mathrm{N}=2^{3}$ was conducted, a regression equation was obtained, the adequacy of this model was proved by methods of mathematical statistics. It is determined that an increase in the size of the input raw material reduces the percentage ratio of the crushed stone output with dimensions of $5-20 \mathrm{~mm}$, and an increase in the rotation speed of the crusher rotor, on the contrary, contributes to an increase in the crushed stone output. Using this equation, you can determine the degree of influence of each factor under investigation on the final result, which is necessary to optimize the process.
\end{abstract}

\section{Introduction}

As you know [1, 2], crushed stone is an inorganic, most often granular, rather loose material, with large grains, more than $5 \mathrm{~mm}$, according to European standards and norms, it is higher than $3 \mathrm{~mm}$. In general, crushed stone production consists of three stages: extraction of raw materials, crushing, and sorting by fractions.

Crushed stone is obtained quite simply by crushing various rocks, such as gravel, boulders, and various simultaneously mined interfering rocks or waste from nonconditioning.

Crushers of different designs are used to produce crushed stone: roller, cone, cheek crushers [3-6].

Roll plants crush stone as a result of its crushing. Their structure is based on two horizontal shafts arranged in parallel. They rotate in opposite directions. The feed material passes between the shafts and is crushed into several parts. The working surface of the

\footnotetext{
* Corresponding author: nigisha.s@mail.ru,
} 
shafts may be corrugated, smooth, or toothed. The latter is especially effective for crushing large pieces of rock.

Cone machines also work on the principle of crush. The main working element of such crushers is a cone rigidly fixed on the shaft. Its lower part is placed in an eccentric sleeve, which drives an electric motor. A movable cone is installed in a fixed conical bowl. When switched on, it performs complex rotational movements, as a result of which the movable and stationary parts periodically approach and are removed, crushing the rock caught between them. Such mechanisms are productive but are very heavy and complex to maintain [7-10].

In cheek crushers, the stone is also crushed according to the crushing principle between two corrugated plates. Both or one of them can be movable. Rock crushing is performed during the approach of cheeks. Such devices are considered simple and easy to maintain, but produce an uneven product in size, are characterized by rapid wear, and tend to clog the working space with a rock.

The centrifugal impact method of crushing non-solid stone materials has been known for a long time and has recently found increasing use. All known today, industrial centrifugal impact crushers have one accelerator and work on the principle of "stone on stone" with a vertical shaft. Such crushers were first used by Barmac Associates (New Zealand) in the 70s. XX century [11].

In most tasks, the crushing and grinding equipment uses the principle of destroying the stone-to-stone material. The use of a self-fouling solution allows you to significantly reduce the cost of quickly worn elements. Destruction occurs due to impact on the accumulated layer of material located in the zone of lining and mutual impact of particles.

The purpose of the present study is to determine experimentally the effect of various factors on the percentage of crushed stone yields produced locally in a vertical impact crusher.

\section{Materials and Methods}

Materials. The gravel of deposit Korakhon Bukhara region of the Republic of Uzbekistan with dimensions of 30-35 $\mathrm{mm}$ is used as initial raw material.

Method for determining the fractional composition of crushed stone. To determine the grain composition of individual fractions of crushed stone, sieves with round or square holes on round or square shells with a diameter or side of at least $300 \mathrm{~mm}$ were used. Standard set of screens for crushed stone (gravel) shall include a screen with square holes measuring $1.25 \mathrm{~mm}$ according to State standard GOST 8269.0-97 and a screen with round holes with diameters $2.5 ; 5(3) ; 7.5 ; 10 ; 12.5 ; 15 ; 17.5 ; 20 ; 22.5 ; 25 ; 30 ; 40 ; 50 ; 60 ; 70$ (80) $\mathrm{mm}$ $[12,13]$.

When determining the quality index of the mixture of fractions, each fraction is tested separately, and the weighted average value of the determined index is determined following the content of the fraction in the mixture by the formula:

$$
X=\frac{x_{1} a_{1}+x_{2} a_{2}+\cdots .+x_{i} a_{i}}{a_{1}+a_{2}+\cdots+a_{i}}
$$

$x_{1}, x_{2}, \ldots x_{i}$ are values of the determined key figure; $a_{1}, a_{2}, \ldots, a_{i}$ is the content of this fraction, $\%$.

Method for determining grain size composition. The grain composition of the crushed stone was determined by sowing a sample on a standard set of sieves.

Description of the experimental plant. The crushed stone crushing process was investigated in a laboratory centrifugal vertical impact crusher, consisting of the following 
parts (Fig 1): feed pipe; housing cover; accelerator; acceleration ribs; armor; electric motor; V-belt transmission.

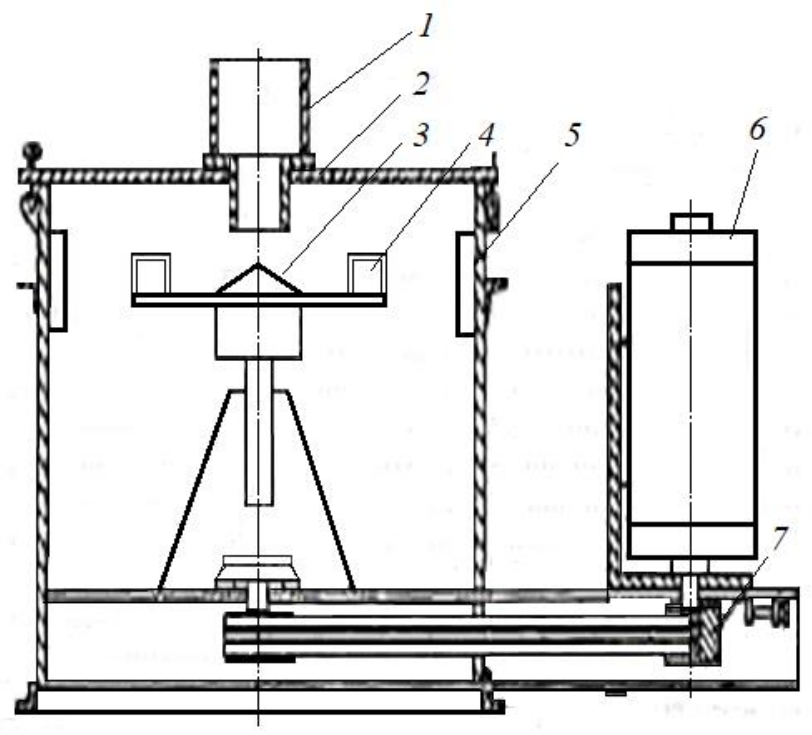

Fig. 1. Laboratory centrifugal vertical impact crusher: 1 is supply nozzle; 2 is housing cover; 3 is accelerator; 4 are acceleration ribs; 5 is armor; 6 is electric motor; 7 is V-belt transmission.

Principle of laboratory crusher operation. Before starting operation, the accelerator installed in the crusher is rotated at a nominal or lower speed. It is gradually supplied with an available small bulk material, which forms a protective layer of self-fouling on the acceleration blades of the accelerator. The moment of the final formation of the protective layer of the accelerator with sand is determined by the fact that sand appears in the discharge hole of the crusher. Then gravel is supplied, containing pieces with a size of $30 \ldots 35 \mathrm{~mm}$. Its movement takes place in the internal channels of the accelerator along with the previously formed layer of sand. The remaining areas (accelerator surfaces not protected by self-fouling) are protected by wear-resistant removable parts made of special materials. This is the edge of the window, the annular plates of the accelerator, and its central zone where the power is supplied.

On the periphery of the crushing chamber, there is a horizontal annular platform - a reflecting surface, which consists of angular baffle plates installed on the annular shell. On this surface, the groundmass forms a surface with a natural slope angle. Pieces of gravel are crushed mainly on this conical reflective surface when they collide with a layer of selffouling. A significant part of the fragments is reflected upward from it. When they fall under the influence of gravity, they re-collide with the flow from the accelerator. Exit the product shall consist of sand with dimensions of $0 . . .5 \mathrm{~mm}$ (concentration in \%) and crushed stone with dimensions of $5 . .20 \mathrm{~mm}$ (concentration in \%).

The effectiveness of the impact interaction depends on the average size of the gravels entering the crusher and the distance between the edge of the accelerator and the surface of the reflective surface. In addition, the crushing effect depends on the rotation speed of the crusher rotor.

Controls and auxiliary equipment. Table dial scales according to the State standard 29329 or laboratory scales according to State standard 24104. Drying cabinet. Sieves and wire round calibers with holes corresponding to nominal grain sizes of this fraction: $1.25 ; 0.5(+)$; as well as 2.5 and $1.25 \mathrm{~mm}$. 
Method for preparing and conducting experiments. A laboratory sample dried to a constant weight is used for the test.

The sample was screened manually through sieves with holes of different sizes, collected sequentially into a column starting from below, from a sieve with holes of the smallest size. At the same time, the thickness of the crushed stone layer on each sieve should not exceed the largest size of crushed stone grains.

The sieving duration is such that no more than $0.1 \%$ of the total weight of the sieved sample passes through each sieve for 1 minute during control intensive manual shaking.

Sieving is considered complete if there is no drop in crushed stone grains.

Method processing of test results are based on the sieving results, the private residue on each sieve $a_{i}, \%$, was determined by the formula [12]:

$$
a_{i}=\frac{m_{i}}{m} 100
$$

$m_{i}$ is the weight of the residue in this sieve, $\mathrm{g} ; m$ is sample weight, $\mathrm{g}$.

The total residues on each sieve were determined as a percentage of the sample weight, equal to the sum of the private residues on the sieve and all sieves with large hole sizes. Scattering of unsorted crushed stone and sand and gravel mixture is carried out using a complete set of standard sieves.

Method for determining the influencing factors on the crushed stone crushing process. A factor is a measured variable that takes at some point in time a certain value and affects the subject of the study $[14,15,16]$.

A factor variation interval is a number (its own for each factor), the addition of which to the main level gives the upper level of the factor, and subtraction - the lower.

For ease of calculation of regression coefficients, all factors during the full factor experiment vary at two levels corresponding to the values of the encoded variables +1 and 1.

The number of experiments of the full factor experiment is determined by the expression $[15,16,17]$ :

$$
N=2^{n}(1)
$$

$n$ is a number of factors.

Based on the above, as the main influencing factors on the crushed stone crushing process, we take:

1. Average incoming raw material size, $d_{e}(\mathrm{~m})$;

2. Crusher rotor speed, $n(\mathrm{rpm})$;

3. Distance between accelerator and annular platform, $l(\mathrm{~m})$.

As the output parameter of $Y$ (out), we take the concentration of crushed stone with a size of 5-20 $\mathrm{mm}$ in the output (\%).

Centrifugal vertical impact crusher.

Method of experimental design. The experimental scheme for solving problems generally consists of the following stages: experiment planning; conducting an experiment; verifying reproducibility; obtaining a mathematical model of an object with checking the statistical significance of polynomial coefficients; checking the adequacy of the mathematical model.

The plan provides for the implementation of all possible experiments, the conditions of which correspond to any combination of values of the investigated factors when they change at only two levels: upper $x_{i}^{+}$lower $x_{i}^{-}-[5,6,7,8,9]$. 
By conducting preliminary experiments, they determined the boundary values of the influencing factors:

The first factor is the incoming size of the raw material, $d_{e}(\mathrm{~m})$ : lower level $x_{1 \min }=0,03$ $0,035 \mathrm{~m}, x_{\text {lmin.mid }}=0.0325 \mathrm{~m}$; we take the upper level $x_{\text {lmax }}=0.035-0.04 \mathrm{~m}$; we accept $x_{\text {Imax.mid. }}=0.0375 \mathrm{~m}$.

The second factor is the rotation speed of the crusher rotor, $n$ (rpm): lower level $x_{2 \min }=1250 \mathrm{rpm}$; upper level $-x_{2 \max }=1500 \mathrm{rpm}$.

The third factor is the distance between the accelerator and the ring platform, $l(\mathrm{M})$ : lower level $x_{3 \min }=0.1 \mathrm{~m}$; upper level $x_{3 \max }=0.2 \mathrm{~m}$.

Experiment center:

$$
\mathrm{X}_{i 0}=\frac{x_{i}^{+}+x_{i}^{-}}{2}
$$

corresponds to the zero $\left(x_{i}=0\right)$ level of the magnitude of the factors, from which the ranges of variation $\varepsilon_{\mathrm{i}}$ are calculated:

$$
\varepsilon_{i}=x_{i}^{+}-x_{i 0}=x_{i 0}-x_{i}^{-}
$$

Thus, according to formulae (2) and (3) for the first factor $-X_{10}=0.035 \mathrm{~m}$; variation interval $\varepsilon_{1}=0.0025 \mathrm{~m}$; for the second factor $-X_{20}=1375 \mathrm{rpm}$; variation interval $\varepsilon_{3}=125 \mathrm{rpm}$; for the third factor $-X_{30}=0.15 \mathrm{~m}$; variation interval $\varepsilon_{3}=0.05 \mathrm{~m}$.

The encoded values of the factor are determined by the expression:

$$
X_{i}=\frac{x_{i}-x_{i}^{0}}{\varepsilon_{i}}
$$

$x_{i}$ is a natural value of the $i-$ th factor; $x_{i}^{0}$ is a natural value of the $i$-th factor at the main level; $\varepsilon_{i}$ is variation interval of the $i$-th factor.

\section{Results and Discussion}

Results of conducted experiments are given in Table 1.

As the output of the process, we consider the percentage of crushed stone with

\begin{tabular}{|c|c|c|c|c|c|c|c|c|c|c|c|}
\hline \multirow{2}{*}{$\begin{array}{c}\text { № } \\
\text { experi } \\
\text { ment }\end{array}$} & \multicolumn{3}{|c|}{ Factors } & \multicolumn{4}{|c|}{ Effects of factor interaction } & \multicolumn{3}{|c|}{$\begin{array}{l}\text { Results of } \\
\text { experiments }\end{array}$} & \multirow{2}{*}{$\begin{array}{r}\begin{array}{r}\text { Average } \\
\text { Results }\end{array} \\
\bar{y}_{u}, \%\end{array}$} \\
\hline & $X_{I}$ & $X_{2}$ & $X_{3}$ & $X_{1} X_{2}$ & $X_{1} X_{3}$ & $X_{2} X_{3}$ & $\begin{array}{c}X_{1} X_{2} X \\
3\end{array}$ & $y_{1}$ & $y_{2}$ & $y_{3}$ & \\
\hline 1 & - & - & - & + & + & + & + & 50.1 & 49.2 & 50.2 & 49.83 \\
\hline 2 & + & - & - & - & - & + & + & 47.2 & 47.5 & 48.1 & 47.60 \\
\hline 3 & - & + & - & - & + & - & - & 50.2 & 50.4 & 50.3 & 50.30 \\
\hline 4 & + & + & - & + & - & - & - & 48.3 & 49.4 & 48.2 & 48.63 \\
\hline 5 & - & - & + & + & - & - & - & 50.2 & 49.2 & 49.1 & 49.50 \\
\hline 6 & + & - & + & - & + & - & - & 48.2 & 47.9 & 48.3 & 48.13 \\
\hline 7 & - & + & + & - & - & + & + & 50.1 & 49.2 & 49.5 & 49.60 \\
\hline 8 & + & + & + & + & + & + & + & 51.2 & 51.5 & 51.4 & 51.37 \\
\hline & & & & & & & & & & $\Sigma \bar{y}_{j}$ & 394.97 \\
\hline
\end{tabular}
dimensions of 5-20 $\mathrm{mm}-y, \%$.

Tab.1. Results of conducted experiments according to scheme $N=2^{3}$. 
For each series of parallel experiments, the arithmetic mean of the response function [59] was found:

$$
\vec{y}_{j}=\frac{1}{k} \sum_{i=1}^{k} y_{j i}
$$

$k$ is number of parallel tests conducted under the same conditions; $j$ is experience number $(j=1,2, \ldots, N) ; i$ is parallel experience number $(i=1,2, \ldots, k)$.

Based on the results of a three-factor experiment, we will compose an equation in which, in addition to linear terms, there will be a term that takes into account the effect of paired inter-factor interaction. The regression equation, in this case, has the following form:

$$
y=b_{0}+b_{1} X_{1}+b_{2} X_{2}+b_{3} X_{3}+b_{12} X_{1} X_{2}+b_{13} X_{1} X_{3}+b_{23} X_{2} X_{3}+b_{123} X_{1} X_{2} X_{3}
$$

The variance estimate for each series of parallel experiments was then calculated by the formula [15-18]:

$$
S_{j}^{2}=\frac{1}{k-1} \sum_{i=1}^{k}\left(y_{j i}-\vec{y}_{j}\right)^{2}
$$

The variance estimate for our case is $S_{j}^{2}=1.6133$

Error of experience is determined by formula:

$$
S_{j}=\sqrt{S_{j}^{2}}
$$

For our case, it is equal to $S_{j}=1.27$.

To check the reproducibility of experiments, we find the ratio of the largest of the variance estimates to the sum of all variance estimates (the calculated value of the Cochran criterion) $[15,19,20]$ :

$$
G_{p}=\frac{\max S_{j}^{2}}{\sum_{j=1}^{N} S_{j}^{2}} .
$$

The calculated value of the Cochran criterion is:

$$
G_{\mathrm{p}}=\frac{0,4433}{1.6133}=0.275
$$

Tabulated values of the Kohren criterion $G_{T}$ are given in [17, Appendix 4]. To find $G_{T}$, you need to know the significance level of $p$ and the number of degrees of freedom $f$ associated with each of them, and $f=k-1, k$-is the number of parallel experiments.

For our case, the significance level is $p=0.05$, and the number of degrees of freedom is $f=k-1=3-1=2$. Then the tabular value of the Cochran criterion is $G_{T}=0.7679$.

Based on the results of the calculations, the condition is met:

$$
G_{p} \leq G_{T}
$$

These experiments are considered reproducible, and the estimates of variances are considered homogeneous. 
If it is established that the estimates of the variances are homogeneous, then the estimate of the variance of the reproducibility of the experiment is calculated by the formula $[15,17]$ :

$$
S_{y}^{2}=\frac{1}{N} \sum_{j=1}^{N} S_{j}^{2}
$$

The estimate of the variance of the reproducibility of the experiment is $S_{y}^{2}=0.2017$

Based on the full factor experiment, the coefficients of the regression equation (6) are determined by the formulas [7-9]:

$$
\begin{gathered}
b_{0}=\frac{1}{N} \sum_{j=1}^{N} \vec{y}_{j} . \\
b_{i}=\frac{1}{N} \sum_{j=1}^{N} X_{J i} \vec{y}_{j} . \\
b_{i m}=\frac{1}{N} \sum_{j=1}^{N} X_{J i} X_{j m} \vec{y}_{j} .
\end{gathered}
$$

The results of the calculations are shown in Table.2.

Table.2. Results of calculations of the coefficients of the regression equation.

\begin{tabular}{|c|c|c|c|c|c|c|c|}
\hline$b_{0}$ & $b_{1}$ & $b_{2}$ & $b_{3}$ & $b_{12}$ & $b_{13}$ & $b_{23}$ & $b_{123}$ \\
\hline 49.371 & -0.438 & 0.604 & 0.279 & 0.463 & 0.538 & 0.2292 & 0.321 \\
\hline
\end{tabular}

Some of the regression coefficients may be negligible-insignificant. To determine whether the coefficient is significant or not, you must first calculate the estimate of the variance with which it is located.

The mean square deviation of the coefficients:

$$
S_{\text {coef }}=\sqrt{\frac{S_{y}^{2}}{N(k-1)}}
$$

In our case: $S_{\text {coef }}=0.1123$

The calculated value of the Stiudent's criterion is determined by the formula:

$$
t_{c r}=\frac{\left|b_{j}\right|}{S_{\text {coef }}}
$$

From the Stiudent's distribution tables $[15,17]$ for the number of degrees of freedom $N(k-1)=8 \cdot 2=16$ at the significance level $\alpha=0.05$, we find $t_{c r}=2.85$.

The absolute value of the coefficient for determining the Stiudent's criterion is calculated by the formula (6):

$$
\left|b_{j}\right|=t_{c r} \cdot S_{\text {coef }}=2.85 \cdot 0.1123=0.32 \text {. }
$$

Comparing the obtained value of 0.32 with the coefficients of the regression equation (see Table.2), we get that all the coefficients are greater in absolute value $\left|b_{j}\right|$, except $b_{3}=0.279$ and $b_{23}=0.2292 \mathrm{~b}$. Therefore, all coefficients except $b_{3}$ and $b_{23}$ are significant.

Thus, the coefficients $b_{3}$ and $b_{23}$ are excluded from the regression equation. 
Then the regression equation (6) has the form:

$$
y=49.371-0.438 X_{1}+0.604 X_{2}+0.463 X_{1} X_{2}+0.538 X_{1} X_{3}+0.321 X_{1} X_{2} X_{3}
$$

The adequacy of the obtained regression equation is checked using the Fisher criterion $\mathrm{F}$ $[15,17]$ :

$$
F=\frac{S_{r v}^{2}}{S_{y}^{2}}
$$

$S_{r v}^{2}$ is the residual variance calculated by the formula:

$$
S_{r v}^{2}=\frac{\sum_{i=1}^{N}\left(y_{i}^{\ni}-y_{i}^{\mathrm{p}}\right)^{2}}{N-L}
$$

$y_{i}^{\ni}$ and $y_{i}^{\mathrm{p}}$ are the experimental and calculated values of the response function obtained in the $i$-experiment; $L$ is the number of significant coefficients in the regression equation.

If the calculated value of the Fisher criterion $F_{c a l}$ is not less than the tabular $\mathrm{F}$ of the table, then the resulting regression equation adequately describes the experiment.

For our case, $S_{r v}^{2}=0.174$.

At the significance level $\alpha=0.05$ and the degrees of freedom of the numerator $f_{l}=N(k$ $1)=8 \cdot 2=16$ and the denominator $f_{2}=N-n-1=8-3-1=4 \quad F_{\text {table }}=5.32[15,17]$.

The calculated value of the Fisher criterion according to the formula (3.16) $F_{\text {cal.val }}=0.86$.

So, $F_{\text {cal }}=0.86<F_{\text {table }}=5.32$. Based on this, we can conclude that the resulting model (7) adequately describes the process.

\section{Conclusions}

The resulting mathematical model (7) can be interpreted as follows:

1. The percentage of crushed stone with dimensions of $5-20 \mathrm{~mm}$ in the crushing process is $49.371 \%$, with the size of the incoming raw material of $0.035 \mathrm{~m}$, the rotor speed of $1375 \mathrm{rpm}$, and the distance between the accelerator and the ring platform of $0.15 \mathrm{~m}$.

2. The rotation speed of the crusher rotor (factor $X_{2}$ ) has a greater effect on the concentration of crushed stone with a size of 5-20 $\mathrm{mm}$ at the output than the average size of the incoming raw material (factor $X_{l}$ ).

3. Increasing the size of the input raw material reduces the percentage of crushed stone output with dimensions of 5-20 mm, and increasing the rotation speed of the crusher rotor, on the contrary, contributes to increasing the crushed stone output with dimensions of 5-20 $\mathrm{mm}$. The influence of the distance between the accelerator and the ring platform on the output is insignificant.

4. The obtained regression equation allows determining with sufficient accuracy the percentage ratio of crushed stone exit with the size of 5-20 $\mathrm{mm}$ in the analyzed range of change of factors. Using this equation, you can determine the degree of influence of each factor under investigation on the final result, which is necessary to optimize the process. 


\section{References}

1. My business. Crushed stone production as a business: list of equipment, description of manufacturing technology. [Internet]. https://moybiznes.org/ [updated 2021 Feb 5; cited 2021 Mar 3]. Available from: https://moybiznes.org/proizvodstvo-schebnya. (2021)

2. Akabirova L.Kh., Khamroeva M.F. Grinding of solids. General basics. Academy scientific and methodological journal. \#1 (64) (2021).

3. How to make crushed stone. [Internet]. "Taxi Pesok" Ltd, https://taxi-pesok.ru/ [updated 2021 Feb 2; cited 2021 Mar 5]. Available from: https:/taxipesok.ru/stati/kak-izgotavlivayut-shcheben..(2021)

4. Ladaev N.M. Efficiency of grinding machines / N.M. Ladaev, L.A. Dmitrieva, F.A. Vaganov. Bulletin of the Scientific and Industrial Society. "Alev-V" -Moscow, (10) .pp.79-80. (2006).

5. Borshchev V. Ya. Equipment for grinding materials: crushers and mills. Tambov: publishing house of the Tambov State Technical University, p 75. (2004).

6. Sidenko P.M. Grinding in the chemical industry. Moscow, (2009).

7. Turaev S.B. Analysis of the design of solid material grinders. 4th International Scientific and Practical Conference "Innovation, Quality and Service in Engineering and Technology", Kursk. pp. 191-192, (2014).

8. Vaganov F.A. Investigation of the process of grinding dry and wet materials and the development of impact mills. Abstract of dissertation for the degree of candidate of technical sciences, Ivanovo (2010)

9. Akabirova L.Kh., Khamidov B.T., Dosumov Sh.R. Investigation of the process of grinding materials based on a mathematical model. Universum-Technical sciences international journal. 10 (67) (2019).

10. Tuz A.A., Sanaeva G.N., Prorokov A.E., Bogatikov V.N., Kulakov A.G. Automatic control systems for a closed-cycle wet grinding unit based on fuzzy models. "Science". 8, (2) (2016) DOI: 10.15862 / 93TVN (2016).

11. Lisica A. Centrifugal impact crusher: history and modernity. [Internet]. Moscow, Russia. [updated 2020 Apr 2; cited 2021 Mar 5]. Available from: https://studfile.net/preview/430874/. (2021)

12. RF Committee on Architecture and Construction. GOST 8269.0-97 Interstate Standard. Crushed stone and gravel from dense rocks and industrial waste for construction work. Methods of physical and mechanical tests. [Internet]. Moscow, Russia. [updated 2019 Sep 1; cited 2021 Mar 18]. Available from: http://docs.cntd.ru/document/1200003066. (2021)

13. Technique for processing bulk materials. V.Ya. Borshchev, V.N. Dolgunin, G.S. Kormiltsin, A.N. Plotnikov. Tambov: Publishing house of Tamb. state tech. un-that, $p$ 40. (2000).

14. Grachev Yu.P., Plaksin Yu. M. Mathematical methods of planning the experiment. DeLee print, p 296. (2005).

15. Sokolovskaya I.Yu. Full factor experiment. NGAVT, p 36. (2010).

16. Gafurov K.Kh., Ibragimov U.M., Fayziev Sh.I. Statistical-mathematical model of the process of extraction of pumpkin seeds by CO2-extraction. Austrian Journal of Technical and Natural Sciences. (1-2) pp.59-63. DOI: https://doi.org/10.29013/AJT17-1.2-59-63 (2017).

17. Derkanosova N. M., Zhuravlev A. A., Sorokina I. A. Modeling and optimization of food production processes. VGTA, p 196. (2011).

18. Xamxanov K.M. Basics of experiment planning. Ulan-Ude, p 50. (2001). 
19. Fayziev Sh.I., Abidov K.Z., Gafurov K.Kh. Experimental study of the technological process of $\mathrm{CO}_{2}$-extraction of ingredients from plant raw materials. Universum: technical sciences. 8 (77). URL: https://7universum.com/ru/tech/archive/item/10635 (2020).

20. Makarichev Yu.A., Ivannikov Yu.N. Methods of experiment planning and data processing. Samara. State Technician. un-t, p131. (2016). 